\title{
Das Immunsystem - wie entsteht antitumorale Immunität?
}

\author{
Yuri Sankawa \\ Stuttgart, Deutschland
}

\section{Organisation des Immunsystems - ein Überblick}

Das menschliche Immunsystem dient in erster Linie der Überwachung der Gewebehomöostase, der Abwehr von Krankheitserregern sowie der Eliminierung von geschädigten Zellen. Eine der wichtigsten Eigenschaften ist dabei die Fähigkeit, eigene und fremde Zellen anhand von Antigenen zu erkennen und voneinander zu unterscheiden. Damit ist das Immunsystem nicht nur in der Lage, Abwehrleistungen gegen exogene Krankheitserreger wie Parasiten und pathogene Mikroorganismen auszuführen, sondern es kann auch eigene, veränderte bzw. entartete Körperzellen im Sinne der Immunüberwachung erkennen und eliminieren (tumor immunosurveillance hypothesis). Das übergeordnete Ziel der Unterscheidung zwischen «selbst» und «fremd» besteht letztlich auch in der Wahrung der Einzigartigkeit und chemischen Individualität eines Menschen [1,2].

\section{Angeborene und adaptive Immunität}

Grundsätzlich werden zwei Formen der Immunreaktion unterschieden: Die angeborene, unspezifische Immunantwort (angeborenes Immunsystem) richtet sich relativ wahllos gegen Krankheitserreger und wehrt den Großteil aller Infektionsereignisse im Laufe des Lebens ab. Zu den Bestandteilen des angeborenen Immunsystems zählen unter anderem Monozyten, Makrophagen, Granulozyten sowie natürliche Killerzellen (NK-Zellen), die auf die Eliminierung von Infektionserregern oder Tumorzellen spezialisiert sind und eine erste, schnelle Vorhut der immunologischen Abwehr bilden. Demgegenüber ist die erworbene Immunantwort (adaptives Immunsystem) «spezifisch», da sie mithilfe von T- und B-Lymphozyten auf einzelne Antigene reagieren kann. Sie hat sich erst im Laufe der Evolution bei Wirbeltieren entwickelt und zeichnet sich unter anderem durch ein «immunologisches Ge- dächtnis» aus, das es dem Immunsystem ermöglicht, sich ein Antigen zu merken, mit dem es bereits Kontakt hatte: Nach einer erstmaligen Infektion bleiben T- und B-Zellen erhalten, die bei einer zweiten Infektion mit diesem Erreger schneller reagieren und zur Expansion antigenspezifischer T- und BZellen führen. Dieser Prozess wird mit jeder neuen Antigenexposition schneller und effektiver [3,4].

Beide Arten der Immunantwort bestehen aus zellulären sowie humoralen (von lat. humor = Flüssigkeit) Komponenten. Während von den zellulären Bestandteilen der angeborenen Immunantwort vor allem Phagozyten und natürliche Killerzellen (NK-Zellen) von Bedeutung sind, stehen bei der erworbenen Immunantwort Subpopulationen der T-Lymphozyten (T-Zellen) wie CD4 $4^{+}$T-Helfer-Lymphozyten (T-Helferzellen) oder $\mathrm{CD}^{+}$zytotoxische T-Lymphozyten (CTL) im Vordergrund [2]. Während die T-Zellen Antigene im Sinne einer zellvermittelten Immunantwort direkt erkennen können, sind im humoralen Teil der erworbenen Immunantwort B-Lymphozyten (B-Zellen) für die Produktion von Antikörpern zuständig.

\section{Spezifität, Diversität und immunologisches Gedächtnis}

Neben der Fähigkeit, zwischen «selbst» und «fremd» zu unterscheiden, gehören auch die Spezifität und Diversität der Immunreaktionen sowie das immunologische Gedächtnis zu den zentralen Eigenschaften des Immunsystems [2]. Dabei sind die Lymphozyten auf ihrer Oberfläche mit Antigenrezeptoren ausgestattet, die nur für ein einziges Antigen spezifisch sind (Spezifität). Ermöglicht wird diese Rezeptorspezifität über einen genetischen Polymorphismus, demzufolge die Antigenbindungsstelle nicht von einem einzelnen Gen, sondern von mehreren Gensegmenten kodiert wird. Mithilfe der sogenannten somatischen Rekombination, die nur in Lymphozyten stattfindet, ist die effiziente Bildung von Millionen ver-

\section{KARGER}

Fax +497614520714

Information@Karger.com

www.karger.com (c) 2014 S. Karger GmbH, Freiburg

2296-5270/14/3717-0002\$39.50/0 
Abb. 1. Checkpoint-Rezeptoren als neue Zielstrukturen der Immunonkologie (modifiziert nach [20]).

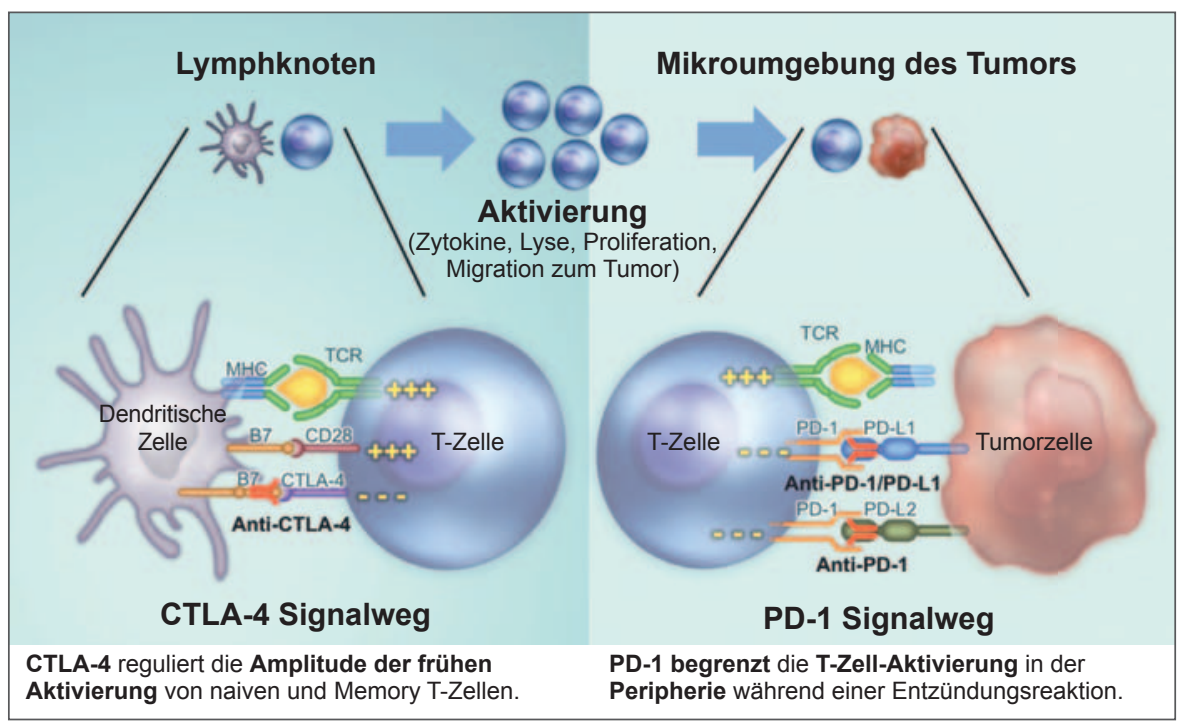

schiedener Antikörper mit unterschiedlichen Spezifitäten möglich (Diversität)

\section{Entstehung des immunologischen Gedächtnisses}

B-Zellen können nach der Bindung eines spezifischen Antigens dieses über Endozytose aufnehmen, verdauen und die Peptidfragmente über MHC-Moleküle der Klasse II auf ihrer Oberfläche präsentieren. Im peripheren lymphatischen $\mathrm{Ge}$ webe treffen sie auf antigenaktivierte Th2-Helferzellen, die mit den B-Lymphozyten interagieren, was letztendlich zur Produktion und Ausschüttung von spezifischen Antikörpern führt. Die aktivierten B-Zellen beginnen sich zu teilen, wandeln sich in Plasmazellen um und produzieren spezifische Antikörper gegen das Antigen [5]. Ein Teil dieser antigenspezifischen Zellen wird in Gedächtniszellen umgewandelt, ruht zum Teil über Jahre im Knochenmark oder anderen Geweben und ermöglicht es dem Organismus, sich bei einem erneuten Kontakt an das Pathogen zu erinnern und schneller bzw. gezielter zu reagieren als beim ersten Kontakt (immunologisches Gedächtnis).

\section{Tumorantigene und Erkennung von Tumorzellen}

Sowohl T-Zell-vermittelte als auch humorale (B-Zell-vermittelte) Immunantworten können Tumorzellen gegenüber spontan auftreten. Dabei bieten Tumorzellen Merkmale, die sie - wenn auch in unterschiedlich starker Ausprägung - von gesunden, normalen Körperzellen unterscheiden. Manchmal sind diese Unterschiede allerdings so geringfügig ausgeprägt dass sie dem Immunsystem entgehen können. Zusätzlich können sich Tumorzellen aktiv gegen die Erkennungsmechanismen des Immunsystems wehren bzw. ihnen entkommen (sogenannter «Tumor Escape») [2].

Im Hinblick auf immuntherapeutische Strategien sind tumorassoziierte Antigene (TAA) von besonderem Interesse. Sie resultieren letztlich aus der Dysregulierung, Mutation oder epigenetisch bedingten Veränderung von Wirtsgenen der Körperzellen: Dem Immunsystem bieten sie die Möglichkeit, diese zu erkennen, und damit eine Angriffsfläche. So können CD ${ }^{+}$CTL die TAA in Verbindung mit MHC-I-Molekülen erkennen und potenziell maligne Zellen angreifen. Im Rahmen der Aktivierung von CTL entstehen auch tumorinfiltrierende Lymphozyten (TIL), die bei der Beseitigung maligner Tumorzellen besonders effektiv und z.B. beim malignen Melanom regelmäßig nachweisbar sind.

\section{Tumorimmunologie:}

\section{Grundlagen der Immunonkologie}

Doch viele der TAA ähneln Selbstantigenen so weit, dass das Immunsystem sie nicht als fremd erkennen bzw. nur schwache Immunantworten auslösen kann. Es gibt auch zahlreiche Beispiele für Tumorzellen, die eine nur verminderte oder sogar fehlende Expression von MHC-I-Molekülen aufweisen und auf diese Weise die Antigenpräsentation weniger wahrscheinlich machen. Die hohe Instabilität und Dysregulation des Tumorgenoms kann schließlich zu einem Verlust von Epitopen führen und die Entwicklung immunresistenter Phänotypen begünstigen $[6,7]$.

\section{Immunevasive Strategien des Tumors}

$\mathrm{Zu}$ den zahlreichen Strategien, die Tumoren im Rahmen komplexer molekularer Mechanismen entwickeln können, um der Immunreaktion zu entkommen (Immunevasion), zählen neben dem Antigenverlust unter anderem auch die Aktivierung anti-apoptotischer Mechanismen sowie Mechanismen zur Herabregulierung der zellvermittelten Immunität (Induktion von Toleranz). Dabei können Tumoren sogar ihr Mikromilieu manipulieren und mediatorvermittelte immunsuppressive Netzwerke errichten, die dem Tumor sein Wachstum und 
Abb. 2. Immunsystem und Krebs: Der Prozess des Immunoediting (modifiziert nach [21]).

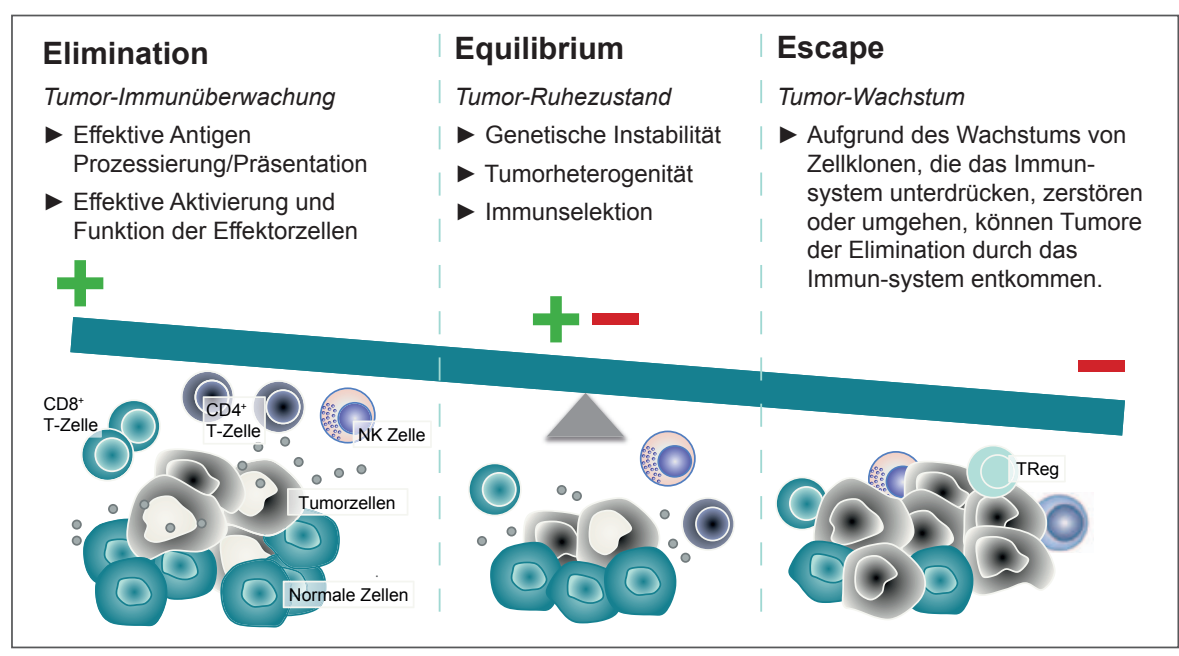

die Progression erleichtern [8]. Beispielsweise können tumorspezifische T-Zellen außer Kraft gesetzt werden, indem der Tumor die Expression sogenannter negativ immunregulatorischer Moleküle beeinflusst.

\section{Immun-Checkpoint-Moleküle - negative Regulatoren der T-Zell-Antwort}

Als das derzeit am besten charakterisierte, negative immunregulatorische Molekül für die T-Zell-Antwort gilt der Proteinrezeptor CTLA-4 (cytotoxic T lymphocyte antigen 4). Neben den Isoformen B7.1 (CD80) und B7.2 (CD86) auf antigenpräsentierenden Zellen stehen CD28 und CTLA-4 auf der Oberfläche von T-Zellen als die wichtigsten Moleküle an der Spitze einer immunologischen Signalkaskade, die der Regulierung der T-Zell-Aktivierung und Immuntoleranz dient [9].

CTLA-4 wird normalerweise in nur sehr niedriger Konzentration auf der Zelloberfläche von T-Zellen exprimiert. Nach erfolgreicher T-Zell-Aktivierung, für die zwei Signale notwendig sind (1.T-Zell-Rezeptor reagiert mit MHC plus Antigen; 2. Costimulierendes Signal durch die Bindung von B7.1 oder B7.2 an CD28) wird der inhibitorische Rezeptor CTLA-4 auf der Zelloberfläche hochreguliert [10]. Nach der T-Zell-Aktivierung bleibt CTLA-4 für gewöhnlich 2-3 Tage hochreguliert [11]. Durch die Ligandenbindung wird das zweite Signal unterbrochen, das T-Zellen benötigen, um eine vollständige Aktivierung aufrechtzuerhalten, sodass ein wirkungsvolles «Abschalten» der T-Zellen zustande kommt [3, 11]. Im Ergebnis reagiert die T-Zell-Population nicht mehr und wird anerg [3]. Die Bedeutung von CTLA-4 bei der Aufrechterhaltung der peripheren Toleranz zeigt sich nicht zuletzt bei Autoimmunerkrankungen, die mit CTLA-4-Polymorphismen assoziiert sind. Auch im Tiermodell entwickelten z.B. CTLA-4-defiziente Mäuse lymphoproliferative Störungen mit unkontrollierter T-Zell-Proliferation und Gewebsinfiltration [12,13].

Einen weiteren negativen Immunregulator der T-Zell-Aktivität repräsentiert der ebenfalls auf T-Zellen exprimierte
Rezeptor PD-1 (programmed cell death 1), der als Transmembran-Rezeptor wie CTLA-4 zur CD28-Immunglobulin-Superfamilie gehört. Im Gegensatz zu CTLA-4 wird PD-1 auf bereits aktivierten T-Effektorzellen exprimiert. Die über dieses Immun-Checkpoint-Molekül ausgelöste Signalkaskade führt zu Apoptose und Herabregulierung der T-Zell-Effektorfunktionen. Der Hauptligand PD-L1 gilt als vorherrschender Mediator der T-Zell-Hemmung und wird verstärkt auf antigenpräsentierenden Zellen, aber auch auf verschiedenen Tumorzelloberflächen exprimiert (Abb. 1). Eine Interaktion zwischen PD-1 und PD-L1 bewirkt eine verminderte Zytokinproduktion und T-Zell-Proliferation und findet in erster Linie im Mikromilieu des Tumors statt [14-16].

\section{Immunoediting - verhängnisvolle Interaktion zwischen} Tumor und Immunsystem

Ein Konzept, das die Interaktion zwischen Immunabwehr und Tumoren beschreibt und Immunreaktionen zum Teil für die Entwicklung und Prägung eines Malignoms mitverantwortlich macht, ist die sogenannte Immunoediting-Hypothese [17]. Dabei werden drei Phasen unterschieden (Abb. 2): In der ersten Phase, der Eliminationsphase, erkennt das Immunsystem maligne transformierte Zellen und zerstört sie, bevor der Tumor klinisch manifest werden kann. In dieser Phase funktioniert die Tumorüberwachung durch das Immunsystem noch, wobei das angeborene und das adaptive Immunsystem miteinander kooperieren. Zur anschließenden Gleichgewichtsphase (Equilibrium) kommt es, wenn bestimmte Tumorzellvarianten der Elimination entkommen. Aufgrund eines dynamischen Gleichgewichts zwischen Tumor und Immunsystem kann das Tumorwachstum aber noch durch das adaptive Immunsystem gehemmt werden.

In diesem Zusammenhang wird auch der Begriff der «Tumor Dormancy» genutzt, d.h. das Immunsystem kann Tumorzellen bzw. den Tumor in eine Art «Dauerschlaf» festhalten. Setzen sich durch einen Selektionsprozess hingegen 
immer mehr immunresistente Zellvarianten durch, geht die Immunkontrolle verloren und der Tumor kann in der dritten Immunoediting-Phase (Escape) klinisch sichtbar werden [17-19].

\section{Disclosure Statement}

Die Autorin hat am «4. Interdisziplinären Expertenforum» teilgenommen, das von Bristol-Myers Squibb unterstützt wurde.

\section{Literatur}

1 de Visser KE, Eichten A, Coussens LM: Paradoxical roles of the immune system during cancer development. Nat Rev Cancer 2006;6:24-37.

2 Ferenčík M, Rovensky J, Mat'ha V, et al.: Kompendium der Immunologie. Wien, Springer, 2006.

3 Abbas AK, Lichtman AH, Pillai S: Cellular and Molecular Immunology, ed 6. Philadelphia, Saunders Elsevier, 2010.

$\checkmark 4$ Zitvogel L, Kroemer G: The immune response against dying tumor-cells: avoid disaster, achieve cure. Cell Death Differ 2008;15:1-2.

$\checkmark 5$ Rink L, Kruse A, Haase H: Immunologie für Einsteiger. Heidelberg, Spektrum Akademischer Verlag, 2012.

6 Algarra I, Garcia-Lora A, Cabrera T, et al.: The selection of tumor variants with altered expression of classical and nonclassical MHC class I molecules: implications for tumor immune escape. Cancer Immunol Immunother 2004;53:904-910.

7 Chang CC, Ferrone S: Immune selective pressure and HLA class I antigen defects in malignant lesions. Cancer Immunol Immunother 2007;56:227236.

8 Kim R, Emi M, Tanabe K, et al.: Tumor-driven evolution of immunosuppressive networks during malignant progression. Cancer Res 2006;66:55275536. $\checkmark 9$ Peggs KS, Quezada SA, Korman AJ, et al.: Principles and use of anti-CTLA4 antibody in human cancer. Curr Opin Immunol 2006;16:206-213.

10 Chambers CA, Kuhns MS, Egen JG, et al.: CTLA4-mediated inhibition in regulation of T-cell responses: mechanisms and manipulation in tumor immunotherapy. Ann Rev Immunol 2001;19:565594.

11 Morse MA: Technology evaluation: ipilimumab, Medarex/Bristol-Myers Squibb. Curr Opin Mol Ther 2005;7:588-597.

12 Tivol EA, Borriello F, Schweitzer AN, et al.: Loss of CTLA-4 leads to massive lymphoproliferation and fatal multiorgan tissue destruction, revealing a critical negative regulatory role of CTLA-4. Immunity 1995;3:541-547.

13 Kristiansen OP, Larsen ZM, Pociot F: CTLA-4 in autoimmune diseases - a general susceptibility gene to autoimmunity? Genes Immun 2000;1:170184.

14 Blank C, Mackensen A: Contribution of the PD-L1/PD-1 pathway to T-cell exhaustion: an update on implications for chronic infections and tumor evasion. Cancer Immunol Immunother 2007;56:739-745.
15 Hamanishi J, Mandai M, Iwasaki M, et al.: Programmed cell death 1 ligand 1 and tumor-infiltrating CD8+ T-lymphocytes are prognostic factors of human ovarian cancer. Proc Natl Acad Sci USA 2007;104:3360-3365.

16 Nurieva RI, Liu X, Dong CT: Yin-Yang of costimulation: crucial controls of immune tolerance and function. Immunol Rev 2009;229:88-100.

17 Schreiber RD, Old LJ, Smyth MJ: Cancer immunoediting: integrating immunity's roles in cancer suppression and promotion. Science 2011;331: $1565-1570$.

18 Swann JB, Smyth MJ: Immune surveillance of tumors. J Clin Invest 2007;117:1137-1146.

19 Dunn GP, Old LJ, Schreiber RD: The immunobiology of cancer immunosurveillance and immunoediting. Immunity 2004;21:137-48.

20 Wolchok JD, Kluger HM, Callahan MK: Safety and clinical activity of nivolumab (anti-PD-1, BMS936558, ONO-4538) in combination with ipilimumab in patients (pts) with advanced melanoma (MEL). J Clin Oncol 2013;31(suppl):abstr 9012.

21 Vesely MD, Kershaw MH, Schreiber RD, Smyth MJ: Natural innate and adaptive immunity to cancer. Annu Rev Immunol 2011;29:235-271. 